\title{
Perceptions, Attitude, Responses, Knowledge and Emotional Well- being (PARKE) of COVID-19 among students at Newcastle University Medicine Malaysia (NUMed)
}

\author{
Joyce Chzi Ching Chee ${ }^{1}$, Sylvia Wei Wei Kong ${ }^{1}$, Zhi Jing Tan ${ }^{1}$, Yi Khai Lim ${ }^{1}$ Mark S Pearce ${ }^{2}$, Edmund L C Ong ${ }^{3}$ (1) \\ ${ }^{1}$ Newcastle University Medicine Malaysia, Johor, Malaysia, 2 Population Health Sciences Institute, Newcastle University, Newcastle Upon Tyne, UK, 3 \\ Newcastle University Medicine Malaysia, Johor, Malaysia; Population Health Sciences Institute, Newcastle University, Newcastle Upon Tyne, UK \\ Keywords: covid-19, perceptions, attitude, knowledge
}

https://doi.org/10.29392/001c.18960

\section{Journal of Global Health Reports}

Vol. 5, 2021

\begin{abstract}
Background
Adherence to preventative measures designed to mitigate transmission of COVID-19 depends on individual's understanding and perception of COVID-19. The objective of this study was to assess the knowledge, perceptions, behavioural adaptation and psychological well-being related to COVID-19 among students attending Newcastle University Medicine Malaysia.
\end{abstract}

\begin{abstract}
Methods
A cross-sectional study was conducted using convenience sampling of students. The self-administered online questionnaire was sent via email in Google forms format between 18 April and 30 April 2020. The questionnaire focused on sociodemographic, perception, attitude and behavioural responses, knowledge and sources of information and anxiety level.
\end{abstract}

\begin{abstract}
Results
326 university students with mean age of 21.8 (S.D 2.3) participated in this study. More females $(n=236)$ took part in the study than males $(n=90)$. Most students $(80 \%)$ believed that they knew how to protect themselves. More than two-thirds (68\%) of students strongly agreed that COVID-19 was a serious public health issue. Most students (>90\%) practised the recommended measures, except for avoid touching of eyes, nose and mouth with unwashed hands (82\%). Wearing a facemask was positively associated with behavioural uptake in university students.
\end{abstract}

\section{Conclusions}

This study showed a good attitude, behavioural responses, knowledge level and emotional responses among NUMed students towards COVID-19.

In December 2019, a series of pneumonia cases of unknown cause emerged in Wuhan, Hubei, China, with clinical presentations resembling viral pneumonia. Deep sequencing analysis from lower respiratory tract samples indicated a novel coronavirus, which was named SARS-CoV-2 $(2019-n C o V){ }^{1}$ Fatal coronavirus infections that have emerged in the past two decades are Severe Acute Respiratory Syndrome Coronavirus (SARS-CoV) and the Middle East respiratory syndrome coronavirus.

Public health measures including maintaining social distance of at least 1 meter, personal hygiene such as cough etiquette and frequent handwashing currently are main recommended interventions to reduce the transmission of COVID-19 in absence of a vaccine. ${ }^{2}$ Adherence of the general population towards public health measures depends on their understanding and perceptions towards COVID-19 infection. A previous study carried out on perception of pub- lic towards influenza (H7N9) suggested that the main reason hindering protective measures were due to perception amongst the general public that the H7N9 virus has low infectivity. ${ }^{3}$

The objective of this study was to assess the knowledge, perceptions, behavioural adaptation and psychological well-being related to COVID-19, among students attending Newcastle University Medicine Malaysia (NUMed), and to assess how their understanding towards the COVID-19 disease would change their responses towards the disease. Questions on sources of information utilized by students were included to assess their impact on students' knowledge towards this disease. 


\section{METHODS}

A cross-sectional study was conducted using convenience sampling of university students from NUMed. The participating students including medical students from Years One to Five, Biomedical Science students in Years One and Two, and Foundation year students.

The self-administered online questionnaire was sent via email to students in Google forms format through an embedded link in the email between 18 April and 30 April 2020 with an accompanying explanation of the purpose of study. The questionnaire (available from corresponding author) comprised of six sections focused on: sociodemographic; perception, attitude and behavioural responses towards COVID-19; knowledge and sources of information of COVID-19 disease; and anxiety level.

Students were asked if they had carried out protective measures recommended by World Health Organisation (WHO) during the pandemic: handwashing for 20 seconds; avoid touching eyes, nose and mouth with unwashed hands; stay at home when sick or had a cold; cover their mouth when coughing; avoid places where many people gather; and social distancing. ${ }^{2}$

These questions were adapted and modified based on the questionnaires used in previous studies for outbreak of Middle East Respiratory Syndrome (MERS), COVID-19 and WHO Protocol. ${ }^{4-7}$ The questionnaire, written in English, took approximately 15 minutes to complete. Pre-testing of questionnaire was conducted using ten students and they were not included into the main study. The students did not have any prior formal preparations about any specific aspects of COVID-19 apart from an optional lecture on principles of prevention and control of infection before they commenced on their clinical attachment in health care settings.

Informed consent was obtained from the students prior to participation. Questionnaires were completed anonymously to ensure confidentiality. Ethical approval was given by the NUMed Research Ethics Committee and the Newcastle Institutional Review Board. A piloting of questions was undertaken with a sample of students to assess the suitability and understanding of questions asked in the survey.

Data were analysed using SPSS version 26. Descriptive analyses were used to determine the sociodemographic data and responses of students for each section. Pearson's correlation test was utilised to identify correlation between COVID-19 and other diseases. A binary logistic regression analysis was applied to analyse the association between response efficacy and behavioural uptakes, with results expressed as odds ratio (OR) and corresponding 95\% confidence intervals ( $95 \% \mathrm{CI})$. Extra data is available by emailing the corresponding author e.l.c.ong@ncl.ac.uk

\section{PATIENT AND PUBLIC INVOLVEMENT}

The students of NUMed, and public were involved in the consultation of the study. The results of the survey will be presented and shared to the students and staff of NUMed and the public at an open event to be held on the university campus.

\section{RESULTS}

\section{DEMOGRAPHIC DATA}

326 university students with mean age of 21.8 (S.D 2.3) participated in this study. More females $(n=236)$ took part in the study than males $(n=90)$. Most students were Malaysian, of Chinese ethnic origin and from the MBBS programme. During the pandemic, most students were at home with their families $(n=213)$. The demographics and other background details of the study population are shown in Table S1 in the Online Supplementary Document.

\section{PERCEPTION}

Students were asked to rate their perception on severity and likelihood of contracting COVID-19 in comparison to other diseases (Table S2 in the Online Supplementary Document). Severe Acute Respiratory Syndrome (SARS) was perceived to be more serious than COVID-19, however, COVID-19 was seen to be similar to pandemic influenza but more serious than seasonal influenza. Cancer and HIV were rated to be more serious than COVID-19 but the correlation coefficients of severity ratings were small. They were also asked to rate the response efficacy of protective measures and their self-confidence towards preventing COVID-19 infection over a 5-point Likert Scale. Most students (80\%) believed that they knew how to protect themselves from coronavirus and more than half (51\%) thought that avoiding COVID-19 infection in the current situation was easy or very easy. (Table S3 in the Online Supplementary Document).

\section{ATTITUDES}

More than two-third (68\%) of students strongly agreed that COVID-19 was a serious public health issue (Table S3 in the Online Supplementary Document). Most students (82\%) also strongly agreed that education was important in preventing the spread of COVID-19.

Medical students were further asked about the effects of COVID-19 on their clinical rotation. More than half of the medical students (54\%) chose to continue their clinical rotation in a hospital that treats COVID-19 patients. However, most students (90\%) refused to do their clinical rotation in a hospital without a clear COVID-19 infection control isolation policy. (Table S3 in the Online Supplementary Document)

\section{BEHAVIOURAL RESPONSES}

Most students (>90\%) practised the recommended measures during the pandemic except for avoid touching of eyes, nose and mouth with unwashed hand (82\%). Students also indicated they had not used antibiotics (95\%) or had flu vaccination (85\%) to prevent being infected by COVID-19 but they exercised caution when opening mail or parcel (63\%). (Table 1)

\section{KNOWLEDGE AND SOURCES OF INFORMATION}

Students were tested on their knowledge on COVID-19 
Table 1. Measures taken to prevent infection from COVID-19

\begin{tabular}{|l|c|c|}
\hline Behaviours & Yes & No \\
\hline Handwashing for 20 seconds & $295(90 \%)$ & $31(10 \%)$ \\
\hline Avoid touching eyes, nose and mouth with unwashed hands & 267 (82\%) & $59(18 \%)$ \\
\hline Staying home when feeling ill & $314(96 \%)$ & $12(4 \%)$ \\
\hline Covering mouth during cough & $314(96 \%)$ & $12(4 \%)$ \\
\hline Avoid gathering at crowded places & $320(98 \%)$ & $6(2 \%)$ \\
\hline Social distancing & $322(99 \%)$ & $4(1 \%)$ \\
\hline Herbal supplements & $28(9 \%)$ & $298(91 \%)$ \\
\hline Getting flu vaccination & $48(15 \%)$ & $278(85 \%)$ \\
\hline Wearing face mask & $297(91 \%)$ & $29(9 \%)$ \\
\hline Using antibiotics & $15(5 \%)$ & $311(95 \%)$ \\
\hline Using caution when opening mail/ parcel & $206(63 \%)$ & $120(37 \%)$ \\
\hline
\end{tabular}

where one mark was given for each correct answer and total knowledge scores were calculated. Most students demonstrated good or very good knowledge of COVID-19 (78\%) with remaining $22 \%$ demonstrated moderate or poor knowledge.

The most used sources of information were online news portals (45\%) followed by government platforms (41\%) and social media (36\%). In contrast, 64\% participants reported they used printed newspapers the least to obtain information on COVID-19, followed by radio stations (47\%) and healthcare providers (46\%). (Table S4 in the Online Supplementary Document) Students had the greatest trust in government sources (39\%), while a fifth trusted social media the least. (Table S5 in the Online Supplementary Document).

\section{PSYCHOLOGICAL IMPACT}

Of the participating students, 58\% did not report any symptoms of anxiety due to the COVID-19 outbreak. The percentages of students reporting 'mild', 'moderate' and 'severe' anxiety were 22,9 and 11 respectively.

\section{ASSOCIATIONS BETWEEN RESPONSE EFFICACY AND BEHAVIOURAL UPTAKE}

Wearing a facemask and taking traditional medicine were positively associated with behavioural uptake in university students. Most of the students (91\%) wore a facemask to protect themselves from COVID-19 even among those 38\% who did not regard wearing a facemask as an effective measure. Logistic regression analysis showed that students who thought wearing facemasks was ineffective in preventing COVID-19 were less likely to wear a facemask (OR=6.15, $95 \% \mathrm{CI}=2.43$ to $15.57, P<0 \cdot 001)$. Students who believed supplements or traditional medicine were ineffective were also less likely to use them $(\mathrm{OR}=10.44,95 \% \mathrm{CI}=3.77$ to 28.86 , $P<0 \cdot 001)$. Students who thought that frequent handwashing was ineffective were also less likely to practice handwashing, although it is not statistically significant (OR=2.65, 95\% $\mathrm{CI}=0.46$ to $5.95, P=0.44$ ). Despite believing that avoid going out is ineffective to protect against COVID-19, all students with this belief still remain at home $(\mathrm{OR}=0,95 \% \mathrm{CI}=0$, $P=0.99$ ). (Table 2)

\section{DISCUSSION}

This is the first study among university students assessing their perception, attitude, behaviour, knowledge and emotional well-being during the first four months of the COVID-19 pandemic in Malaysia. The main findings showed a good attitude, behavioural responses, knowledge level and emotional responses among NUMed students towards COVID-19.

In comparison to COVID-19, our findings showed that students perceived SARS, cancer, and HIV/AIDS to be more serious, pandemic influenza to be as serious and seasonal influenza to be less serious if contracted. A study done by Kwok et al. demonstrated that SARS was as serious as COVID-19 while pandemic influenza, cancer, HIV/ AIDS were less serious than COVID-19. ${ }^{4}$ Both studies reported seasonal influenza to be less serious than COVID-19. This could be that the study by Kwok et al. was carried out in China which was the epicentre for COVID-19 and SARS during the early stage of outbreak when it was associated with a high case fatality rate. Higher perceived severity of COVID-19 can lead to irrational behaviours of panic buying of personal protective equipment for infection prevention. ${ }^{5}$

Students displayed confidence in protecting themselves against COVID-19 during this pandemic. Qian et al. in China also found that majority of the participants in their study had confidence on taking measures to protect themselves. ${ }^{5}$ The high level of confidence may be linked to experiences learnt from SARS pandemic that had high mortality in Asia. A large-scale study comparing efficacy beliefs two years after the outbreak of SARS demonstrated a more positive efficacy beliefs in Asia than Europe. ${ }^{8}$ High level of self-confidence, together with high risk perception and high response efficacy can motivate adoption of precautionary behaviours. ${ }^{8}$

Students strongly agreed that COVID-19 was a serious public health issue. This perception might have rooted from 
Table 2. Associations between response efficacy and behavioural uptake in reducing COVID-19 infection risk

\begin{tabular}{|c|c|c|c|c|c|c|}
\hline \multirow{3}{*}{$\begin{array}{l}\text { Response efficacy } \\
\text { Frequent handwashing }\end{array}$} & \multicolumn{2}{|c|}{ Behavioral uptake } & \multirow{3}{*}{$\begin{array}{l}\text { OR } \\
1.65\end{array}$} & \multirow{2}{*}{\multicolumn{2}{|c|}{$95 \% \mathrm{Cl}$}} & \multirow{3}{*}{$\begin{array}{c}P \text {-value } \\
0.44\end{array}$} \\
\hline & \multirow[t]{2}{*}{ Yes } & \multirow[t]{2}{*}{ No } & & & & \\
\hline & & & & 0.46 & 5.95 & \\
\hline Effective & 277 & 28 & - & - & - & - \\
\hline Ineffective (Reference) & 18 & 3 & - & - & - & - \\
\hline Total & 295 & 31 & & & & \\
\hline \multicolumn{3}{|l|}{ Wearing face mask } & 6.15 & 2.43 & 15.57 & $<0.001$ \\
\hline Effective & 183 & 6 & - & - & - & - \\
\hline Ineffective (Reference) & 114 & 23 & - & - & - & - \\
\hline Total & 297 & 29 & & & & \\
\hline \multicolumn{3}{|l|}{ Avoid going out } & 0 & 0 & 0 & 0.99 \\
\hline Effective & 298 & 12 & - & - & - & - \\
\hline Ineffective (Reference) & 16 & 0 & - & - & - & - \\
\hline Total & 314 & 12 & & & & \\
\hline \multicolumn{3}{|c|}{ Supplements or traditional medicine } & 10.44 & 3.77 & 28.86 & $<0.001$ \\
\hline Effective & 8 & 11 & - & - & - & - \\
\hline Ineffective (Reference) & 20 & 287 & - & - & - & - \\
\hline Total & 28 & 298 & & & & \\
\hline
\end{tabular}

the high transmissibility of SARS-CoV-2 virus. The basic reproduction number (Ro) is an epidemiologic metric used to describe the contagiousness or transmissibility of infectious agents. An outbreak is expected to continue if the Ro has a value of $>1$ and to end if Ro is $<1$. The $R_{0}$ of SARS-CoV-2 ${ }^{9}$ is around 2.0-2.5, which is higher than the $\mathrm{R}_{0}$ of SARS (1.7 to 1.9$)$ and MERS $(<1) .{ }^{10}$ In response to "flattening the curve", the Malaysian Government enforced the Movement Controlled Order (MCO), equivalent to 'lockdown' in other countries. The effect COVID-19 had on the economy had further highlighted it as a devastating public health issue, with far wider reaching health consequences than just the direct danger of the virus, and subsequent illness, itself. Students also strongly agreed upon the importance of education in preventing the spread of COVID-19. The effectiveness of health education has been explored in previous study. ${ }^{11}$ Wang et al. affirmed that health education effectively improve knowledge on infectious disease and therefore boost uptake of protective behaviours. ${ }^{11}$ Medical students believing in the importance of health education is not unexpected. It remains to be seen whether this view is shared by the general population, or whether a potential increase in heath literacy during the pandemic will have a long-lasting effect to protect against other diseases and future pandemics.

More than half of the medical students would continue their clinical rotation in hospitals that treat COVID-19 patients. This is in contrast to a study in Ecuador that disclosed 58\% medical students had negative attitude towards volunteering at health facilities. ${ }^{12}$ Their reluctance could be attributed to a shortage of personal protective equipment (PPE), lack of guidance and clinical supervision as $91 \%$ students said they did not have appropriate PPE. ${ }^{12}$ Most of the students in our study also agreed that they would not undertake their clinical rotation in a hospital without clear COVID-19 infection control isolation policy. Transmission of COVID-19 from infected patients to healthcare workers has been reported in cases of unprotected patient contact. ${ }^{13}$ WHO published guidelines on prevention of COVID-19 in healthcare workers, ${ }^{14}$ and the guideline has been closely adhered to by Malaysians Ministry of Health (MOH) and the PPE supply chain in Malaysia was never compromised. ${ }^{15}$ All these supportive measures reassured medical students to continue developing their clinical skills despite knowing the deadly threat of COVID-19 to many healthcare workers during this pandemic.

Our findings showed most students adopted the six recommendations by WHO during this pandemic. We attribute these positive behaviours to good knowledge about COVID-19 of the students. Being aware of the mode of transmission of the virus direct them to take up relevant protective behaviours. It is not known whether any of these recommendations were already adopted by students prior to the pandemic. The positive correlation between knowledge levels and behavioural uptakes is consistent with that seen in previous studies. 4,16

Students in this study had, correctly, not taken antibiotics or had the flu vaccine specifically to prevent COVID-19 infection. This demonstrated that our students are well-informed that antibiotics and flu vaccination are not efficacious against COVID-19. In contrast, $21 \%$ participants in a study in Jordan wrongly believed that taking antibiotics will protect them from being infected with COVID-19. ${ }^{17}$ This difference might partly be explained by the difference in prevalence of COVID-19. At the time of study, only one case had been diagnosed in Jordan but there had been 5305 
cases in Malaysia. ${ }^{17,18}$ Increased disease prevalence, generally, attracts more attention to understand the disease. WHO has reinforced that COVID-19 is a virus and antibiotics do not work against viruses. ${ }^{19}$ There is an opportunity during this pandemic for wider health education that antibiotics do not prevent or treat viruses that may help reduce the misuse of antibiotics around the world.

In general, our students demonstrated good level of knowledge on COVID-19. Most students know the modes of transmission, incubation period, symptoms, groups at risk and availability of treatment or vaccine for COVID-19. This is consistent with findings from Alzoubi et al. that reported adequate knowledge level among university students. ${ }^{17}$ This is related to their education level as it correlates positively with knowledge score. Informed individuals are more likely to actively acquire knowledge of infectious disease through various channels. ${ }^{16}$ After the declaration of COVID-19 as a pandemic by WHO, there has been a surge of information through media and health authorities. There has also been a surge of misinformation, primarily through social media. The distrust of social media for health information amongst these students is reassuring.

The common sources of information used by students were online news portal, government resources and social media. Most studies also reported social media and online resources as the two most common information sources. ${ }^{4,17}$ However, participants in study by Alzoubi et al. rated government source as the second least used sources of information because one third of the participants were not generally satisfied with the efforts contributed by government. ${ }^{17}$ In contrast, most Malaysian agreed that government had handled the COVID-19 pandemic well. ${ }^{20}$ During the lockdown period, the National Security Council of Malaysia sent out text messages on daily basis to provide updates on COVID-19 outbreak. Therefore, students trusted government sources the most as government had been playing a proactive role in disseminating information pertaining to COVID-19.

The COVID-19 pandemic did not have significant impact on emotional aspects among the students in our study. More than half of students did not report any symptoms of anxiety. Nonetheless, our study reported higher rates of anxiety among students (42\%) compared to a study carried out in China which revealed only $25 \%$ college students were afflicted with anxiety during the COVID pandemic. ${ }^{21}$ Students' anxiety might have been secondary to the sudden announcement of university closure leading to the switch to online learning, and uncertainty on academic progression especially in final year students which made up the largest proportion in our sample. These results pinpoint the need for prompt educational and psychological support to address concerns arise during pandemic to avoid adverse psychological reactions. Teaching should be continued as soon as possible through online platform and guides should be given to students on how to access those online resources, with additional support given to those struggling with resources, such as computers or internet, or with external commitments such as the care and/or education of other family members.

Interestingly, our study found most students wore facemasks during this period, although some have perceived facemasks as ineffective. This may be due to evidence from previous studies on the effectiveness of facemasks to prevent transmission of respiratory viruses. ${ }^{22}$ WHO initially did not recommend the use of facemasks unless symptomatic, or if taking care of persons infected with COVID-19. ${ }^{23}$ However, on $5^{\text {th }}$ June 2020, WHO updated its guidelines to encourage the general public to wear facemasks while in public areas i.e. taking public transport or during mass gatherings to prevent community transmission. ${ }^{24}$ Timely and consistent information should also be disseminated by the authorities to the public because inaccurate information can lead to misconceptions that result in unnecessary behavioural uptakes. Taking into account online resources are a frequently used source for our students, and the great deal of trust given to government advice, information dissemination in Malaysia can be undertaken through infographics on MOH's portal or the official Facebook page.

The current study has several limitations. This study only involved students from NUMed which mainly comprised a young population with a background in medical sciences, and thus not representative of the general public. Future research should investigate whether the findings from this study can be applied to the diverse population in Malaysia. The sample size in our study $(n=326)$ is also smaller compared to another similar study $(n=592) .{ }^{17}$ Despite a small sample size, we achieved a good response rate of more than $40 \%$. Lastly, there might be some reporting bias due to the self-reporting nature of the survey as students might want to conceal their negative behaviours. The use of online anonymous survey should hopefully have mitigated this potential bias.

\section{CONCLUSIONS}

Our study should provide baseline information for health authorities when developing health promotion programmes targeting student populations. Health promotion programmes can be integrated into future curriculum to strengthen knowledge on infection control, because knowledge amplifies positive behavioural responses. Our study also stressed the importance of accurate and consistent information dissemination between different health authorities to prevent misconception about such infectious diseases that might raise unnecessary behavioural and psychological responses. The best platform to consider for information dissemination, for Malaysia, would be through videos uploaded by health authorities onto social media or online news portal since those were most frequently used by the younger generation. Prompt educational and psychological support should be undertaken by tertiary educational organisations to students to guide them throughout this period of uncertainty, especially those away from their families.

\section{ACKNOWLEDGEMENTS}

Not applicable. 


\section{ETHICS APPROVAL AND CONSENT TO PARTICIPATE}

The study was approved by the Research Management Group of the Newcastle University Medicine Malaysia and all students gave consent for participation in the online study.

\section{CONSENT FOR PUBLICATION}

Not applicable.

\section{AVAILABILITY OF DATA AND MATERIAL}

Please contact corresponding author for data requests

\section{FUNDING}

Not applicable

\section{AUTHORSHIPS CONTRIBUTIONS}

Conceptualization: Ong ELC,

Data curation: Chee JCC, Kong SWW, Tan ZJ, Lim YK, Pearce MS, Ong ELC

Formal analysis: Chee JCC, Kong SWW, Tan ZJ, Lim YK, Pearce MS, Ong ELC
Investigation: Chee JCC, Kong SWW, Tan ZJ, Lim YK, Pearce MS, Ong ELC

Methodology Chee JCC, Kong SWW, Tan ZJ, Lim YK, Pearce MS, Ong ELC

Project administration: Ong ELC

Supervision: Pearce MS, Ong ELC

Writing - original draft: Chee JCC, Kong SWW, ELC Ong

Final draft: Chee JCC, Kong SWW, Tan ZJ, Lim YK, Pearce MS, Ong ELC

All authors read and approved the final manuscript.

\section{COMPETING INTERESTS}

The authors declare that they have no competing interests

\section{CORRESPONDENCE TO}

Dr ELC Ong, MBBS, MSC, FRCP, FRCPI, DTMH

Address: Newcastle University Medicine Malaysia (NUMed Malaysia)

No. 1 Jalan Sarjana 1, Kota Ilmu, EduCity@Iskandar 79200 Iskandar Puteri (formerly Nusajaya), Johor, Malaysia e.l.c.ong@ncl.ac.uk

Submitted: November 01, 2020 GMT, Accepted: January 15, 2021 GMT 


\section{REFERENCES}

1. Cheng ZJ, Shan J. Novel coronavirus: Where we are and what we know. Infection. 2020;48(2):155-163. do $\mathrm{i}: 10.1007 / \mathrm{s} 15010-020-01401-\mathrm{y}$

2. World Health Organisation. Coronavirus disease (COVID-19) advice for the public. Published online 2020. Accessed April 1, 2020. https://www.who.int/em ergencies/diseases/novel-coronavirus-2019/advice-fo r-public

3. Chan EY, Cheng CK, Tam G, Huang Z, Lee P. Knowledge, attitudes, and practices of Hong Kong population towards human A/H7N9 influenza pandemic preparedness, China, 2014. BMC Public Health. 2015;15(1):943. doi:10.1186/s12889-015-224 $\underline{5-9}$

4. Kwok KO, Li KK, Chan HH, et al. Community responses during the early phase of the COVID-19 epidemic in Hong Kong: Risk perception, information exposure and preventive measures. MedRxiv. Published online February 27, 2020. doi:10.1101/202 $\underline{0.02 .26 .20028217}$

5. Qian M, Wu Q, Wu P, et al. Psychological responses, behavioral changes and public perceptions during the early phase of the COVID-19 outbreak in China: A population based cross-sectional survey. medRxiv. Published online February 20, 2020. doi:10.1101/202 $\underline{0.02 .18 .20024448}$

6. Elrggal ME, Karami NA, Rafea B, et al. Evaluation of preparedness of healthcare student volunteers against Middle East respiratory syndrome coronavirus (MERS-CoV) in Makkah, Saudi Arabia: A crosssectional study. J Public Health. 2018;26(6):607-612. d oi:10.1007/s10389-018-0917-5

7. WHO Regional Office for Europe. COVID-19 Snapshot MOnitoring (COSMO Standard): Monitoring knowledge, risk perceptions, preventive behaviours, and public trust in the current coronavirus outbreak WHO standard protocol. PsychArchives. Published online 2020 .

8. de Zwart O, Veldhuijzen IK, Elam G, et al. Perceived threat, risk perception, and efficacy beliefs related to SARS and other (emerging) infectious diseases: Results of an international survey. Int J Behav Med. 2009;16(1):30-40. doi:10.1007/s12529-008-9008-2

9. Diekmann O, Heesterbeek JAP, Metz JAJ. On the definition and the computation of the basic reproduction ratio $\mathrm{R} 0$ in models for infectious diseases in heterogeneous populations. J Math Biol. 1990;28(4):365-382. doi:10.1007/bf00178324
10. Petrosillo N, Viceconte G, Ergonul O, Ippolito G, Petersen E. COVID-19, SARS and MERS: Are they closely related? Clinical Microbiology and Infection. 2020;26(6):729-734. doi:10.1016/j.cmi.2020.03.026

11. Wang M, Han X, Fang H, et al. Impact of health education on knowledge and behaviors toward infectious diseases among students in Gansu Province, China. BioMed Research International. 2018;2018:1-12. doi:10.1155/2018/6397340

12. Eddy LN, Paola SP, Santiago RV, et al. Paradigms about the COVID-19 pandemic: Knowledge, attitudes and practices from medical students. medRxiv. Published online May 26, 2020. doi:10.1101/2020.05.2 $\underline{1.20105858}$

13. Heinzerling A, Stuckey PMJ, Scheuer T, et al. Transmission of COVID-19 to health care personnel during exposures to a hospitalized patient-Solano County, California, February 2020. Published online February 2020.

14. World Health Organisation. Infection Prevention and Control during Health Care When Novel Coronavirus (NCoV) Infection Is Suspected.; 2020. Accessed April 20, 2020. https://www.who.int/publica tions/i/item/10665-331495

15. Jr JK, Chung C. Health Ministry Mass Purchasing PPE, Issues Guidelines for Its Usage. TheStar2020

16. Zhong BL, Luo W, Li HM, et al. Knowledge, attitudes, and practices towards COVID-19 among Chinese residents during the rapid rise period of the COVID-19 outbreak: A quick online cross-sectional survey. Int J Biol Sci. 2020;16(10):1745-1752. doi:10.7 150/ijbs.45221

17. Alzoubi H, Alnawaiseh N, Al-Mnayyis A, Lubad M, Aqel A, Al-Shagahin H. COVID-19-knowledge, attitude and practice among medical and nonmedical University Students in Jordan. J Pure Appl Microbiol. 2020;14(1):17-24. doi:10.22207/jpam.14.1.0 $\underline{4}$

18. Ministry of Health Malaysia. Situasi Terkini 18 April 2020.; 2020. Accessed April 18, 2020. http://covi d-19.moh.gov.my/terkini/042020/situasi-terkini-18-a pril-2020

19. World Health Organisation. Coronavirus Disease (COVID-19) Advice for the Public: Myth Busters.; 2020. https://www.who.int/emergencies/diseases/novel-cor onavirus-2019/advice-for-public/myth-busters 
20. Azlan AA, Hamzah MR, Sern TJ, Ayub SH, Mohamad E. Public knowledge, attitudes and practices towards COVID-19: A cross-sectional study in Malaysia. PLoS ONE. 2020;15(5):e0233668. doi:10.1 371/journal.pone.0233668

21. Cao W, Fang Z, Hou G, et al. The psychological impact of the COVID-19 epidemic on college students in China. Psychiatry Research. 2020;287:112934. doi:1 0.1016/i.psychres.2020.112934
22. Leung NHL, Chu DKW, Shiu EYC, et al. Respiratory virus shedding in exhaled breath and efficacy of face masks. Nat Med. 2020;26(5):676-680. doi:10.1038/s41591-020-0843-2

23. World Health Organisation. Advice on the Use of Masks in the Context of COVID-19. World Health Organisation; 2020.

24. World Health Organization. Advice on the Use of Masks in the Context of COVID-19: Interim Guidance, 5 June 2020. World Health Organization; 2020. 


\section{SUPPLEMENTARY MATERIALS}

\section{Online Supplementary Document}

Download: https://www.joghr.org/article/18960-perceptions-attitude-responses-knowledge-and-emotional-wellbeing-parke-of-covid-19-among-students-at-newcastle-university-medicine-malaysia-num/attachment/50463.docx 